\title{
Compressive Sensing Based Image Compression and Transmission for Noisy Channels
}

\begin{tabular}{l|l|}
\hline Yan Zhang $^{1}$ (iD \\
Suxia Cui \\
Yonghui Wang
\end{tabular}

\begin{abstract}
This paper presents the design of an optimized Compressive Sensing image compression technique for data transmission over noisy mobile wireless channel. The proposed technique is more robust to channel noise. It uses individual measurement driven coding scheme, which facilitates simpler encoder design. The shift of computational burden from encoder to decoder is more suitable for mobile devices applications where computational power and battery life are limited. This paper also presents a novel quantizer which allows the encoder to dynamically adapt to the channel conditions and provides optimum performance.
\end{abstract}

Keywords: Compressive sensing, Coding with feedback, Image transmission, Quantization, Joint source-channel coding.

\section{Contents}

1. Introduction.

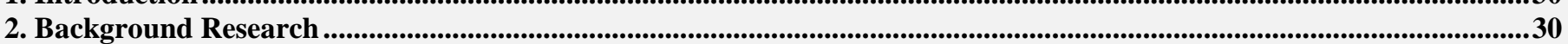

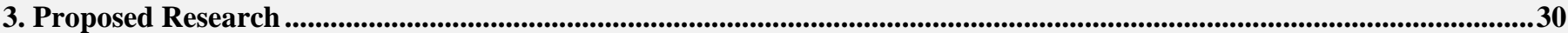

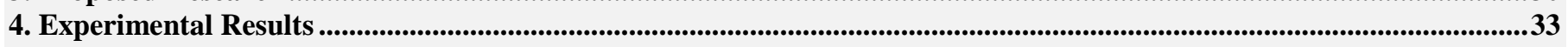

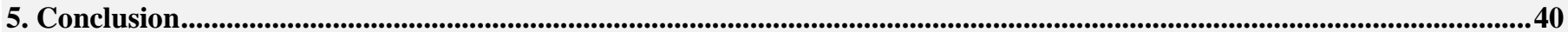

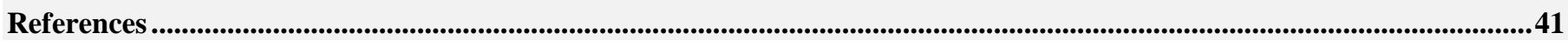

Citation | Yan Zhang; Suxia Cui; Yonghui Wang (2017). Compressive Sensing Based Image Compression and Transmission for Noisy Channels. International Journal of Modern Research in Electrical and Electronic Engineering, 1(1): 29-41.

Licensed: $\quad$ This work is licensed under a Creative Commons Attribution 3.0 License $(\mathrm{oc}) \mathbf{E Y}$

Contribution/Acknowledgement: All authors contributed to the conception and design of the study.

Funding: $\quad$ This work is sponsored by ARO and the United States National Science Foundation (NSF) \#1229744, \#1332566,

Competing Interests: $\quad \# 0930243$, and \#1411260.

Transparency: The authors declare that they have no conflict of interests. The authors confirm that the manuscript is an honest, accurate, and transparent account of the study was reported; that no
vital features of the study have been omitted; and that any discrepancies from the study as planned have been explained.

History:

Ethical:

Publisher: This study follows all ethical practices during writing. Asian Online Journal Publishing Group 


\section{Introduction}

As more and more mobile devices are being used with new innovations to support multimedia applications, e.g, Instagram, Facebook, wireless spectrum is becoming increasingly scarce The evolution of new applications is demanding spectrum available to the increasing mobile users [1]. The design of mobile devices for inclusion of new and evolving multimedia applications faces big challenges due to: a) limited battery capability for power supplies; b) wireless transmission noise and quality; and c) limited processor (CPU and GPU) capability on mobile devices. Thus data compression for wireless networks is becoming more and more important. This research takes image as a sample multimedia data type to explore the necessary improvement. Traditional image transmission utilizes popular compression standards to reduce data size first. For example, Joint Photographic Experts Group (JPEG) uses blockbased Discrete Cosine Transform (DCT) to provide high compression rate for image coding and the transmission of these coded data. The block-based scheme encounters difficulties of image recovery in dynamically varying noisy channels in wireless [2-4]. Compressive Sensing (CS) technique was initially proposed [5, 6] to enable signals sampled at sub-Nyquist sample rate to be perfectly recovered. For the past decade, CS has been widely adopted in various signal processing areas, but it is not fully explored for applications in wireless networks. In this paper, we present an optimized CS based image transmission scheme over noisy Gaussian and fading channels.

\section{Background Research}

\section{A. Compressive Sensing}

Compressive sensing of images allows sampling at a sub-Nyquist rate onto a random basis and could also be reconstructed to the original image with the condition of sparsity.

The $N$-dimensional signal $x$ is assumed to be $K$-sparse with respect to some orthogonal matrix $V$. The "sampling" of $x$ is a linear transformation by using a matrix $\phi$ to produce a vector $y=\phi x$. Let $\phi$ be an $M$-by- $N$ matrix where $M<<N$, so $y$ has M elements; we call each element of $y$ as a measurement of $x$. The decoder recovers the signal $x$ from $y$ with known $V$ and $\phi[7]$.

\section{B. Image Compression Standards}

JPEG is the most popular still image compression standard. It is the first international standard for still image compression where the encoder and decoder are DCT-based [2]. The DCT achieves transform domain decorrelation on image compression [8]. JPEG2000 uses a more advanced transform-Discrete Wavelet Transform (DWT) - to reach a higher compression ratio.

\section{Network Model}

Current image compression standards, such as JPEG, are highly optimized source coding approach. However, in a real wireless network, it is important to take the channel conditions into consideration to optimize the overall performance. This is more important in mobile wireless networks as transmission errors occur in various forms and mobility itself has an impact on reliability of transmission.

In this research, we first started with a Gaussian noisy channel where the original signal over a channel is subject to additive white Gaussian noise. One can model a channel as follows:

$$
Y_{i}=X_{i}+Z_{i}
$$

Where, $Y_{i}$ is the channel output, $X_{i}$ is the channel input, and $Z_{i}$ is the Gaussian channel noise. Experiments show that, if the channel noise level is low, JPEG coded images can be reconstructed well. However, the reconstruction of JPEG images becomes difficult at high noise levels, where packet loss is frequent and it will significantly downgrade the reconstructed image quality. Thus traditional image coding techniques may not perform well for mobile wireless network applications. CS provides a promising alternative for wireless networks. This paper proposes a CS based image transmission system with novel quantization technique. The optimization of the coding parameters is discussed. The results are compared to JPEG based technique. Besides Gaussian channel, results from Rician and Rayleigh fading channel are also provided for a more convincing conclusion.

\section{Proposed Research}

\section{A. Block Schematic of the Proposed System}

The basic block diagram of the proposed system is shown in Figure-1. It consists of a feedback loop to optimize the image transmission for different channel conditions.

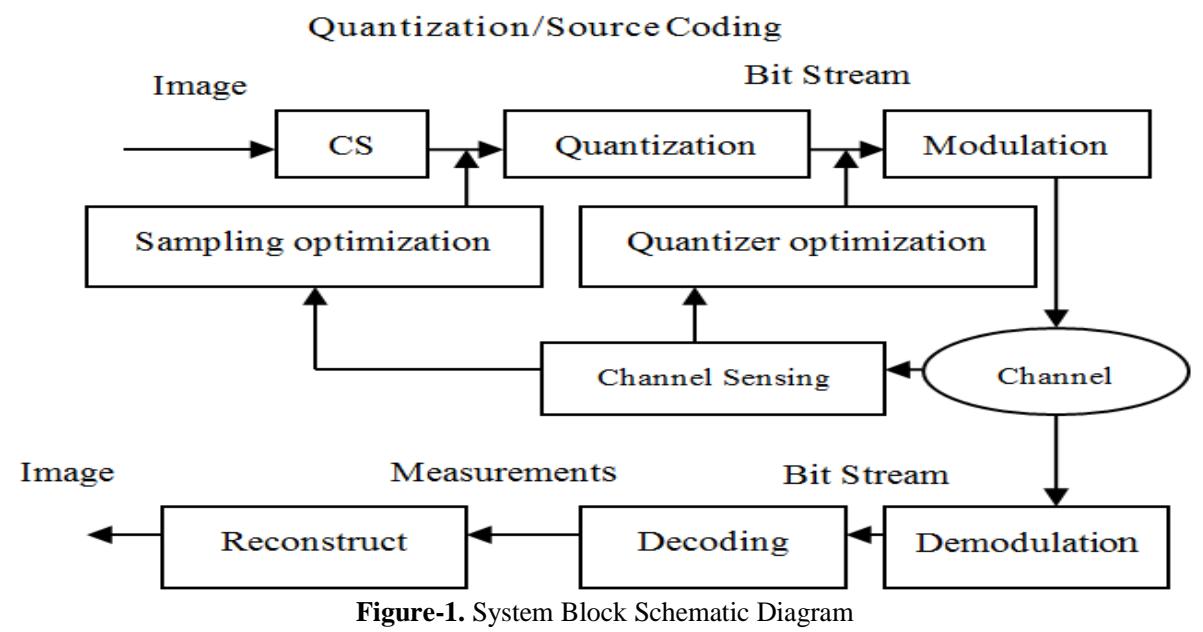


There would be a sensor to sense the channel's conditions. Based on the sensed channel condition, the system determines the optimal sampling rate (percentage of the measurements), and also, the number of bits/measurements to achieve optimized PSNR is selected as part of the quantizer.

\section{B. Recovery Error Calculation}

According to Candès and Terence [5] an image could be near perfectly recovered from the given vectors (measurements). In Candès and Terence [5] the relationship between number of measurements and the error between recovered and original images was discussed. However, the authors only gave a bound of the error. We would need to further discuss a numerical relationship between the number of measurements and recovered signal errors.

\section{Encoder Optimization}

In Candès and Terence [5] it is proved that an image could be recovered from sub-Nyquist samples with the help of CS technology. CS based encoder generates random measurements. Each measurement carries a certain amount of information that can be recovered independently. The more measurements received at the decoder side, the better reconstruction quality can be achieved [9]. We will further discuss how many sub Nyquist samples (subrate) to use to achieve optimal image recovery.

Figure- 2 shows the recovered images which are reconstructed using a variety percentages of measurements. We will see that even by using a small amount of measurements (1\%) it still can roughly recover the whole image. Also, there is no significant difference if we use a larger amount of measurements for the recovery of images.
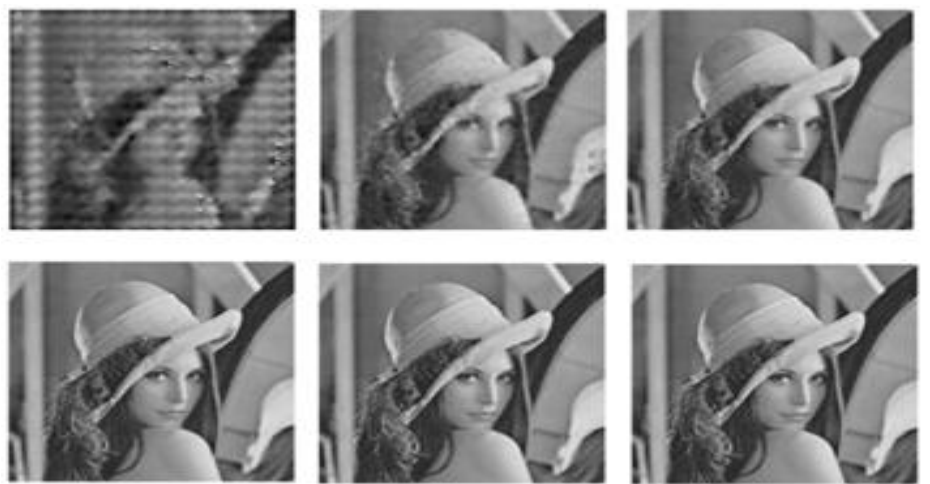

Figure-2. Reconstructed image from sub-Nyquist measurements: from left to right: Percentage of measurements and PSNR: $1 \%, 16.097 \mathrm{~dB}$; $5 \%, 22.272 \mathrm{~dB} ; 10 \%, 28.232 \mathrm{~dB} ; 30 \%, 32.997 \mathrm{~dB} ; 50 \%, 36.5 \mathrm{~dB} ; 90 \%$, $45.159 \mathrm{~dB}$

\section{Measurement Distribution}

Besides the number of measurements, to build an efficient coding system to transmit bit stream, there is a need to optimize the quantizer (source coding). To optimize the quantizer, the statistical properties of the encoded data are investigated. Figures- 3 and -4 show the distribution of measurements and the Normal distribution fit with the same mean and variance.

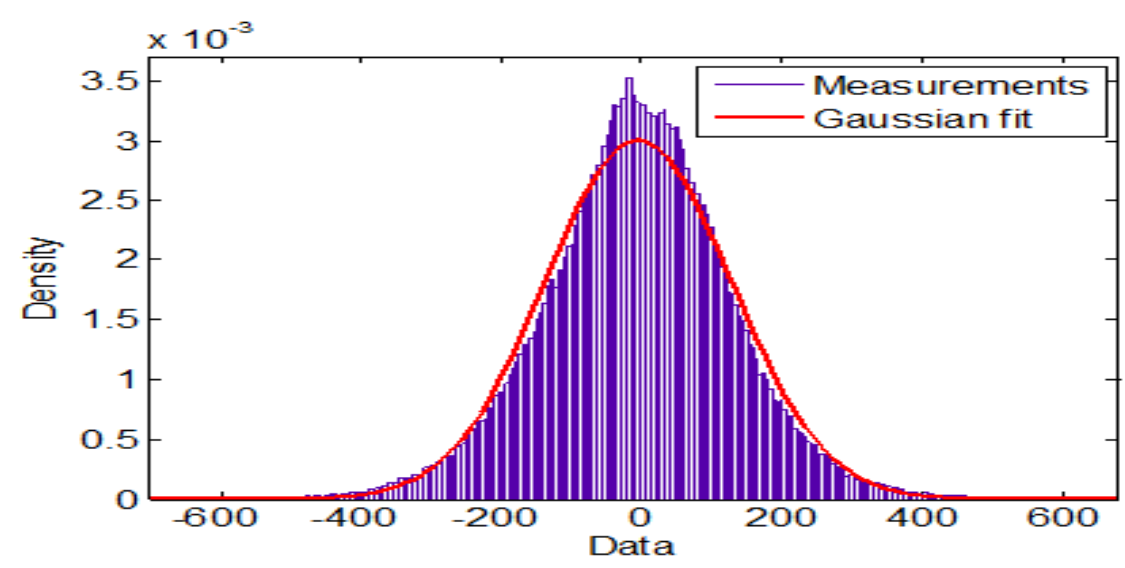

Figure-3. PDF Comparison of Lenna CS measurements with Gaussian distributions

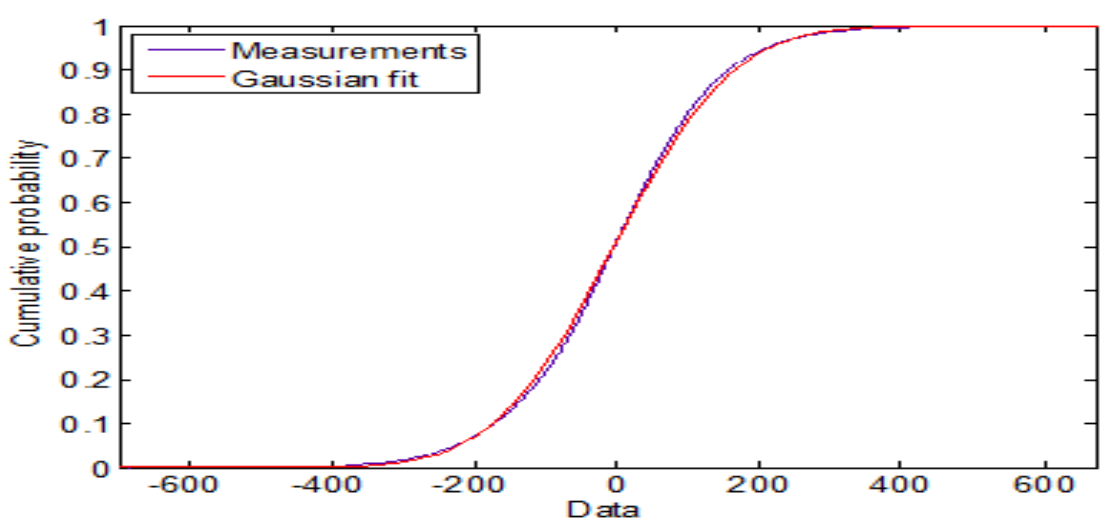

Figure-4. CDF Comparison of Lenna CS measurements with Gaussian distributions 
From the probability density function (PDF) and the cumulative distribution function (CDF), Lenna's CS measurements have the same mean and variance fit with their Gaussian distribution. We could conclude that the distribution of Lenna CS measurements is "Gaussian-like distributed". We have tried many other images and all the CS measurements are distributed like Gaussian distribution. Table-1 lists the mean and standard deviation for various images. It can be observed that the CS measurements are all "Gaussian like distributed" and the parameters (mean and standard deviations) are very close and we could use "standard" parameters to design encoders for the CS measurements without significant errors.

Table-1. Distributions of Image Measurements

\begin{tabular}{l|l|l}
\hline \multicolumn{3}{|c}{ Table-1. Distributions of Image Measurements } \\
\hline Lenna & mean & standard deviation \\
\hline Barbara & 2.1778 & 132.94 \\
\hline Goldhill & 2.0366 & 129.46 \\
\hline Cameraman & 1.9533 & 122.51 \\
\hline Living room & 2.0436 & 133.56 \\
\hline Peppers & 2.1013 & 128.77 \\
\hline Lake & 2.1017 & 128.63 \\
\hline Mandril & 2.3582 & 140.58 \\
\hline Pirate & 2.2965 & 134.52 \\
\hline Walkbridge & 1.8724 & 121.36 \\
\hline Aerial & 1.8657 & 126.37 \\
\hline Couple & 2.5442 & 147.62 \\
\hline Elaine & 2.2913 & 129.50 \\
\hline Tank & 2.3562 & 143.90 \\
\hline Truck & 2.3369 & 135.10 \\
\hline Moonsurface & 1.8414 & 110.46 \\
\hline Women_darkhair & 2.2027 & 130.71 \\
\hline On Average & 1.8425 & 124.21 \\
\hline standard deviation of means:0.2212 & 131.81 \\
\hline standard deviation of standard deviations : 9.3521 \\
\hline
\end{tabular}

\section{E. CDF Based Quantizer}

Here we propose a quantizer (source coder), which is based on the CDF of CS measurements. Suppose the distribution's pdf function is $f(x)$, if there are $i$ bits per sample, to get a vector of segments $X$, the distribution will need to be divided into $2^{i}$ quantization steps:

Let $n=2^{i}$ and $x=\{x 1, x 2, \ldots ., x(n-1))$, where

$$
\int_{-\infty}^{x 1} f(x) d x=\int_{x 1}^{x 2} f(x) d x=. .=\int_{x(n-1)}^{\infty} f(x) d x=\frac{1}{n}
$$

That is, if we use $x_{i}$ to express the inverse function of cdf,

$$
x_{i}=c d f^{-1}\left(\frac{i}{n}\right)
$$

Also, we have a vector $x_{c}=\left\{x_{c 1}, x_{c 2}, \ldots, x_{c n}\right\}$ as codebook for decoding

Where

$$
\begin{aligned}
\int_{-\infty}^{x c 1} f(x) d x= & \int_{x c 1}^{x 1} f(x) d x=\ldots . . \int_{x i}^{x c(i+1)} f(x) d x=\ldots \ldots . .=\int_{x c n}^{\infty} f(x) d x=\frac{1}{2 n} \\
& -\infty<x_{c 1}<x_{1} ; x_{1}<x_{c 2}<x_{2} ; \ldots \ldots . .
\end{aligned}
$$

Or,

$$
x_{c i}=c d f^{-1}\left(\frac{i}{2 n}\right)
$$

For Gaussian distributed data, if mean $\mu$ and variance $\sigma$ are known, it is easy to implement the quantizer:

Let $\Phi^{-1}$ be the inverse of the standard normal CDF, the quantize function is:

$$
\begin{aligned}
& x_{i}=\mu+\sigma^{*} \Phi^{-1}\left(\frac{i}{n}\right) \\
& x_{c i}=\mu+\sigma^{*} \Phi^{-1}\left(\frac{i}{2 n}\right)
\end{aligned}
$$

Then the total expectation of error square is

$$
\mathrm{D}=\sum E\left(\text { error }^{2}\right)=\sum_{i=0}^{n} \int_{x i}^{x^{x i+1}} f(x) *(x-x c(i+1))^{2} d x
$$

The total error will be equal to the sum of all the areas as shown in Figure 5. 


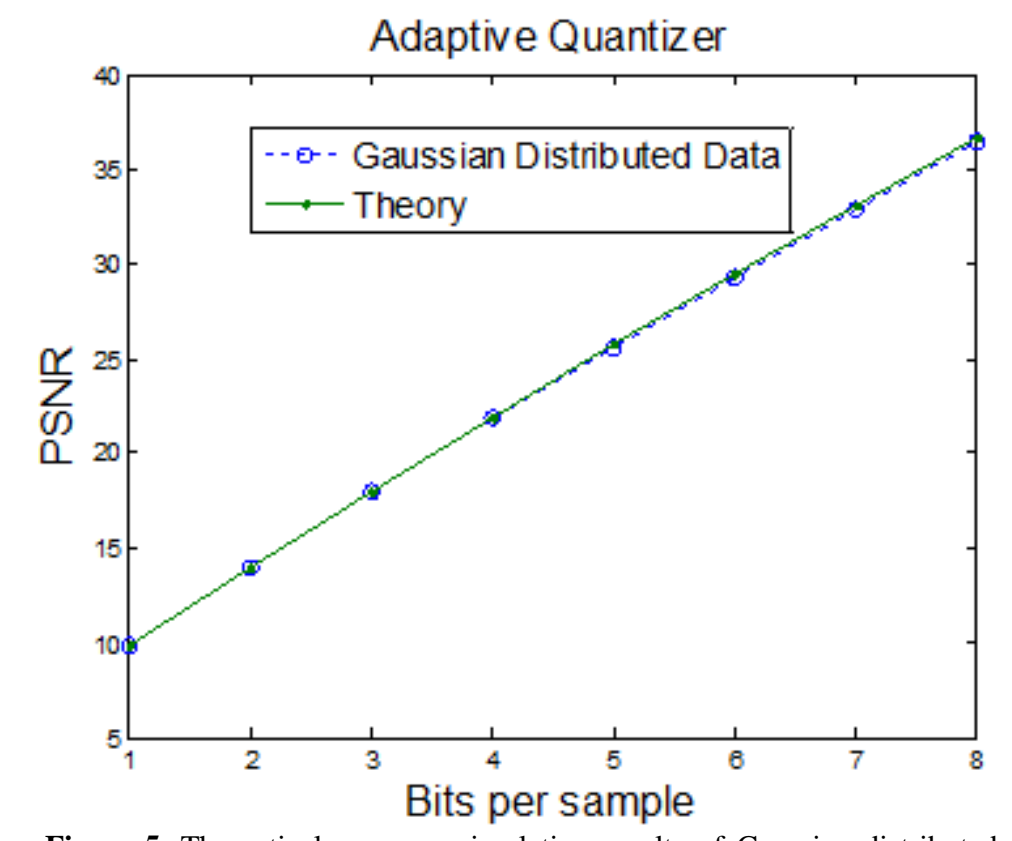

Figure-5. Theoretical error vs. simulation results of Gaussian distributed data without channel noise

\section{F. Advantages of Proposed Coder}

1) Easy to implement: It is only based on the uniform division of CDF, then it is very easy to design.

2) Fast computing and processing: Since the distributions of measurements on different images are very close, it is possible to set a fixed quantizer for fast coding.

3) It has better performance in noisy channels.

\section{G. Gaussian Distributed Sample Analysis}

In an $m \mathrm{~dB}$ signal-noise-ratio (SNR) Gaussian channel, for Binary Phase Shifting Keying (BPSK) modulation [10] the bit error rate would be

$$
\operatorname{Ber}=0.5 * \operatorname{erfc}\left(\sqrt{10^{\frac{m}{10}}}\right)
$$

For a $k$ bits system, the transmitting error calculation is shown below.

There are total of $2^{k}$ areas. Take 3 bits for example, if the first bit is wrong through transmission, '000' would be decoded as ' 100 ' and ' 001 ' would be decoded as ' 101 '.

The total error square is

$$
\operatorname{error}^{2}=B e r * \sum_{i=i}^{k} \sum_{j=1}^{2^{k}}\left(C D F^{-1}\left(\frac{j}{2^{k}}-\frac{1}{2^{k+1}}\right)-C D F^{-1}\left(\frac{j}{2^{k}}-\frac{1}{2^{k+1}}+\frac{1}{2^{i}}\right)\right)^{2}
$$

An example is shown in Fig. 6.

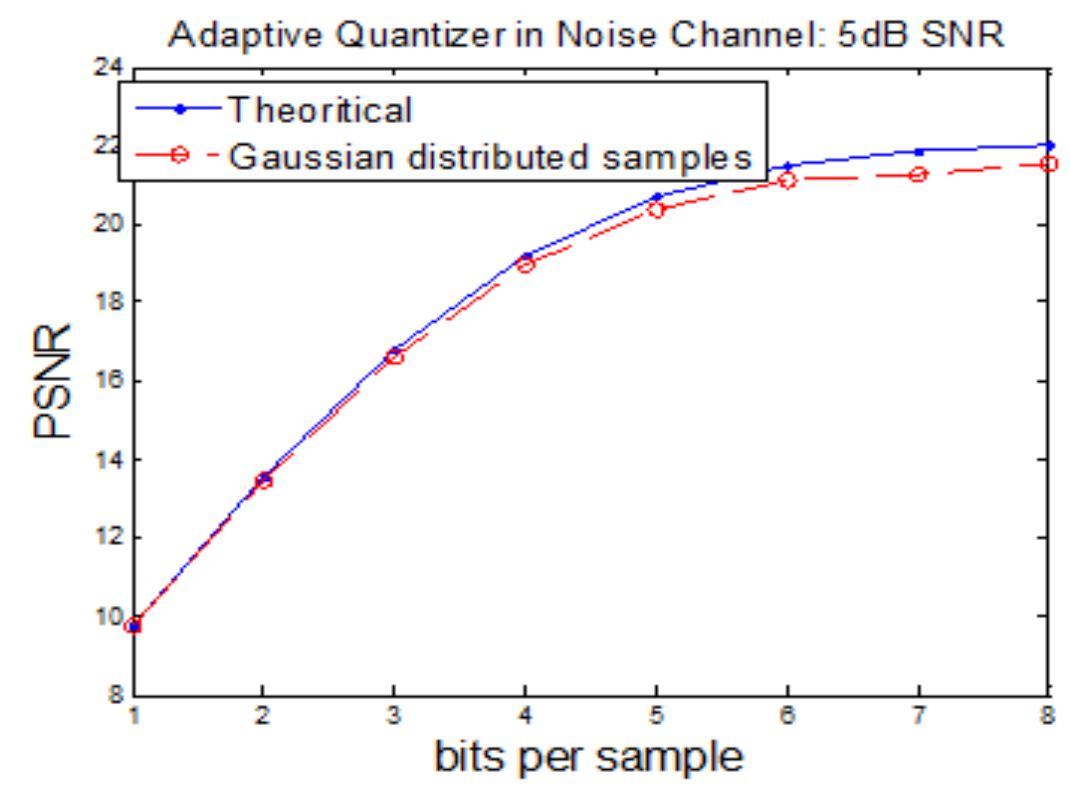

Figure-6. Theoretical error vs. simulation results of Gaussian distributed data $5 \mathrm{~dB}$ Gaussian channel

\section{Experimental Results}

All the following simulation are based on the measurements from 'Lenna', which is $\mathbf{5 1 2} \times \mathbf{5 1 2}$ in gray scale. All the modulation are binay BPSK.

\section{A. Quantizers to be Compared}

In order to analyze the efficiency of our proposed quantizer, two popular quantizers, linear quantizer and Lloyd_Max quantizer, are chosen for comparison. 


\section{1) Linear}

The basic quantizer is the uniform scalar quantizer. A linear quantizer for image measurements has been discussed in Zhang, et al. [9].

\section{2) Lloyd_Max}

S. Lloyd had developed least MSE quantizer for scalar quantization [11]. He also gave the quantization table in Lloyd [11]. However, Lloyd_max quantizer may not be the optimum in noisy channels.

\section{B. Performance in Noisy Channel}

We tested our proposed quantizer along with the linear and Lloyd_Max quantizers in 5dB Gaussian channel and the results are in Figure-7. From this, we can conclude that our CDF based quantizer is better in low dB (high noise) channel (when $\mathrm{SNR}<=8 \mathrm{~dB}$ ) than Lloyd quantizer and linear quantizer.

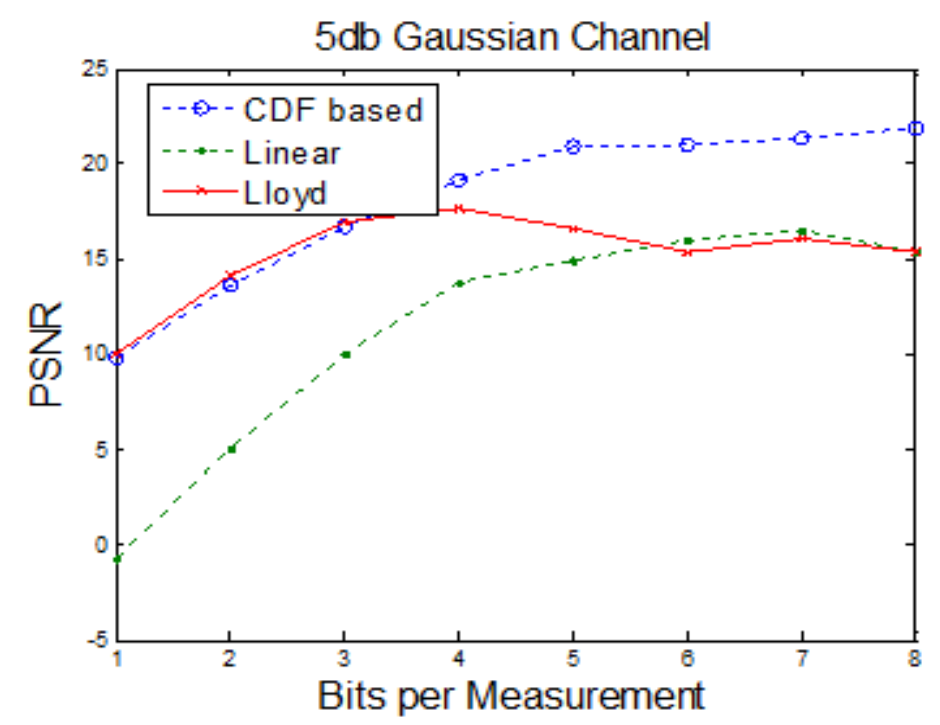

Figure-7. Comparison of 3 quantizers in $5 \mathrm{~dB}$ Gaussian channel

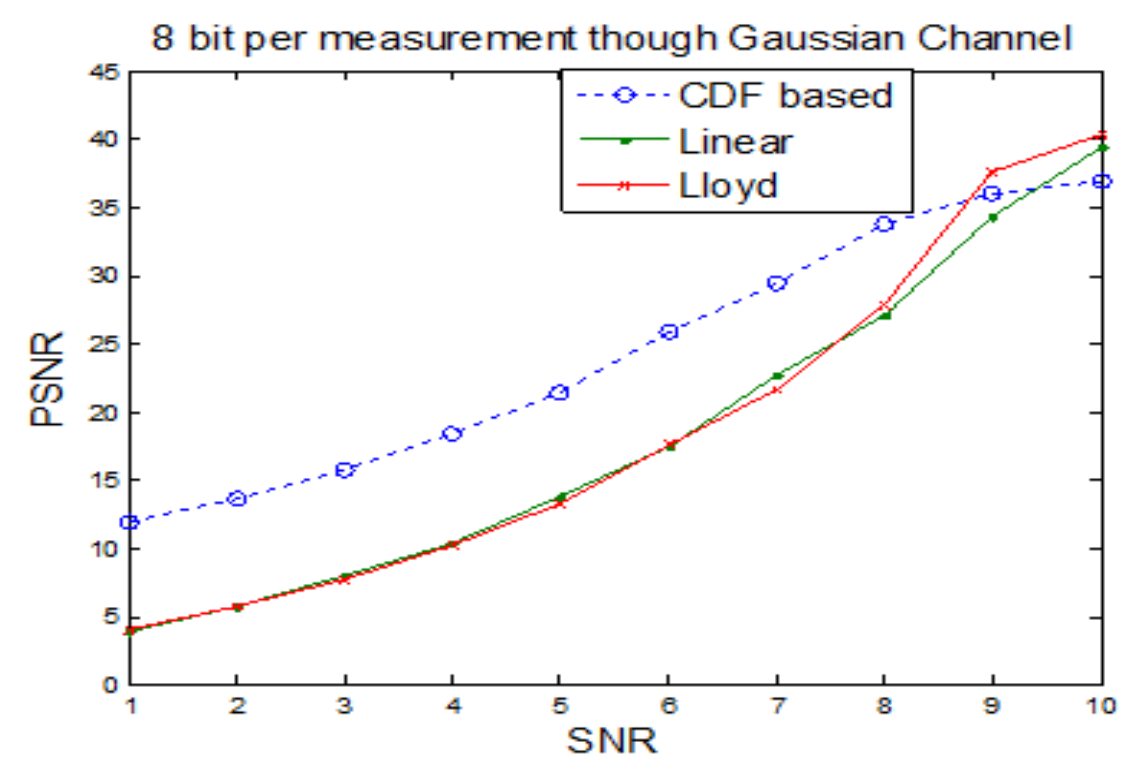

Figure-8. Comparison of 3 quantizers in Gaussian channe with 8 bits/measurement

A brief discussion: Comparison of segments and codebook training sets for Lenna with 3 bits/measurement:

Lloyd segments: [-226.1, -127.1, -51.2, 16.6, 85.1, 167.7, 286.2];

Codebook:[ -284.0, -168.1, -86.05, -16.4, 49.6, 120.6, 214.7, 357.7];

CDF based segments: [-146.1764, -83.9351, 37.3906, 4.2867, 45.9640, 92.5084, 154.7498];

Codebook:[-196.3728, -111.7501, -59.6442, -16.2892, 24.8626, 68.2175, 120.3235, 204.9462].

From above data we can see that our CDF based quantizer is more centralized than Lloyd. Then in the noisy channel, when an error occurs in a bit, this causes less difference than Lloyd quantizer. Thus it outperforms Lloyd quantizer in noisy channels. Please refer Figure-8 for experimental results.

\section{Power Adjustments}

Consider that for mobile devices, power is very limited. We can use less bits and then give each bit more power. Equation (9) presents how . Bit Error Rate (BER) value is calculated for BPSK.

If we change it to $k$ bits encoder (suppose the original was 8 bits), the equation becomes

$$
\operatorname{Ber}(k)=0.5 * \operatorname{erfc}\left(\sqrt{\frac{8}{k} 10^{\frac{m}{10}}}\right)
$$

Figure-9 shows the experimental results validating the theoretical values. 


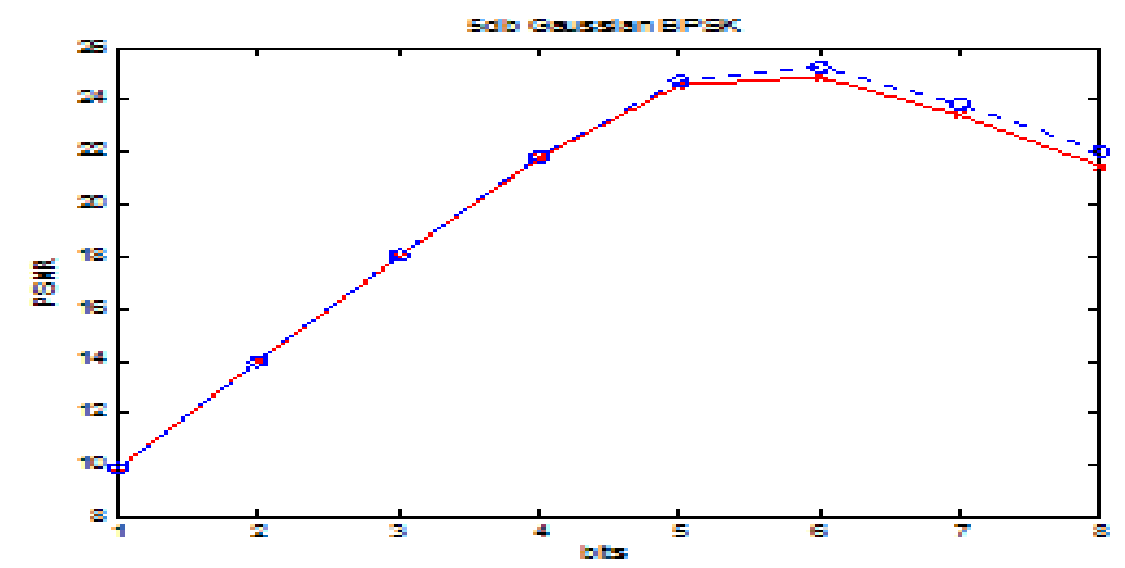

Figure-9. Theoretical error vs. simulation results of Gaussian distributed data in $5 \mathrm{~dB}$ Gaussian channel with power adjusted

Figure-10 shows the PSNR of power adjusted performance in 1-10 dB Gaussian channels. We can conclude that in a Guassian noisy channel, with power adjusted, there is an optimal value of bits/measurements which will achieve the optimized value of PSNR. Table-2 shows the optimal bits/measurements for each channel SNR.

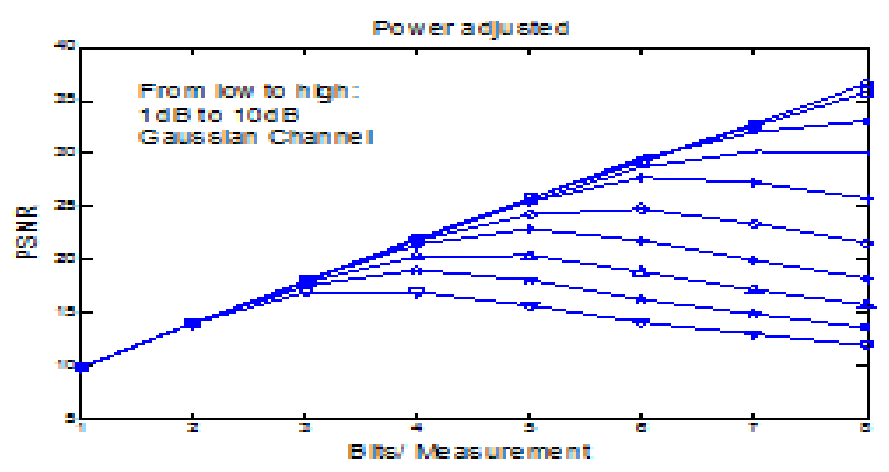

Figure-10. PSNR with bits power adjusted in different channel SNR

Table-2. Optimized bits per measurement in different channel SNR

\begin{tabular}{l|l}
\hline SNR (dB) & Optimal bits/measurement \\
\hline 1 & 4 \\
\hline 2 & 4 \\
\hline 3 & 5 \\
\hline 4 & 5 \\
\hline 5 & 6 \\
\hline 6 & 6 \\
\hline 7 & 7 \\
\hline 8 & 8 \\
\hline 9 & 8 \\
\hline & 8 \\
\hline
\end{tabular}

\section{Subrate vs. Less Bits}

To save bits in transmission, there are trade-offs of how to optimize the system. In CS based coding, the subsample rate is given by the size of an $\mathrm{M}$ by $\mathrm{N}$ matrix. We've discussed the quantization code book and adjustable bit power, so under different channel condition, maintain a high subsample rate with less bits per code or vise versa may lead to different quality for reconstructed images. In this paper, we discussed two choices to further reduce the total bits per compressed image: 1) to reduced measurements / samples of an image (subrate); and 2) to reduce bits/ measurements.

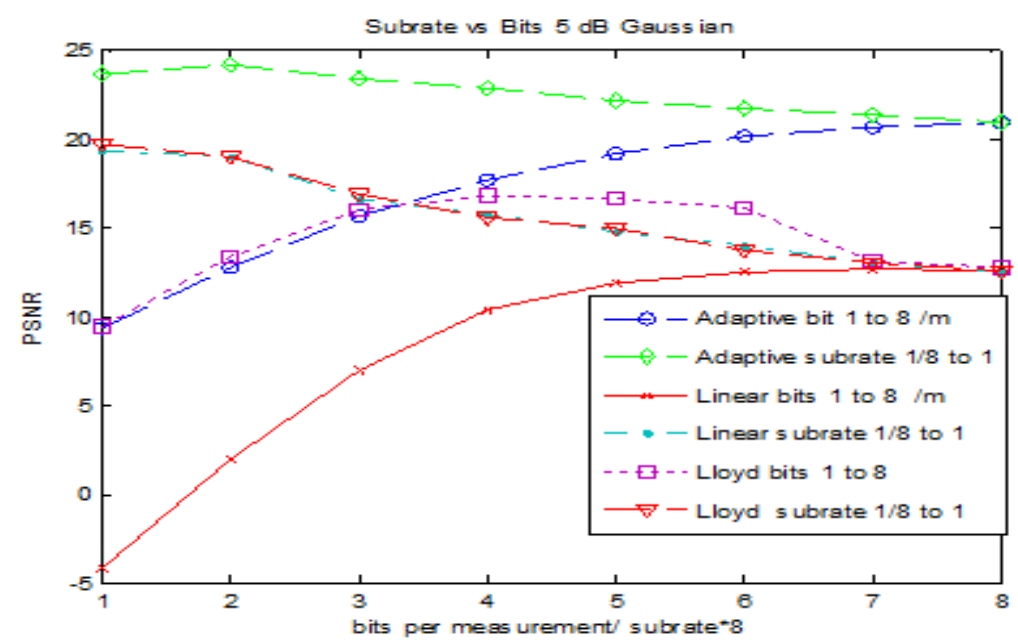

Figure-11. Subrates vs. bits in 5dB Gaussian Channel 


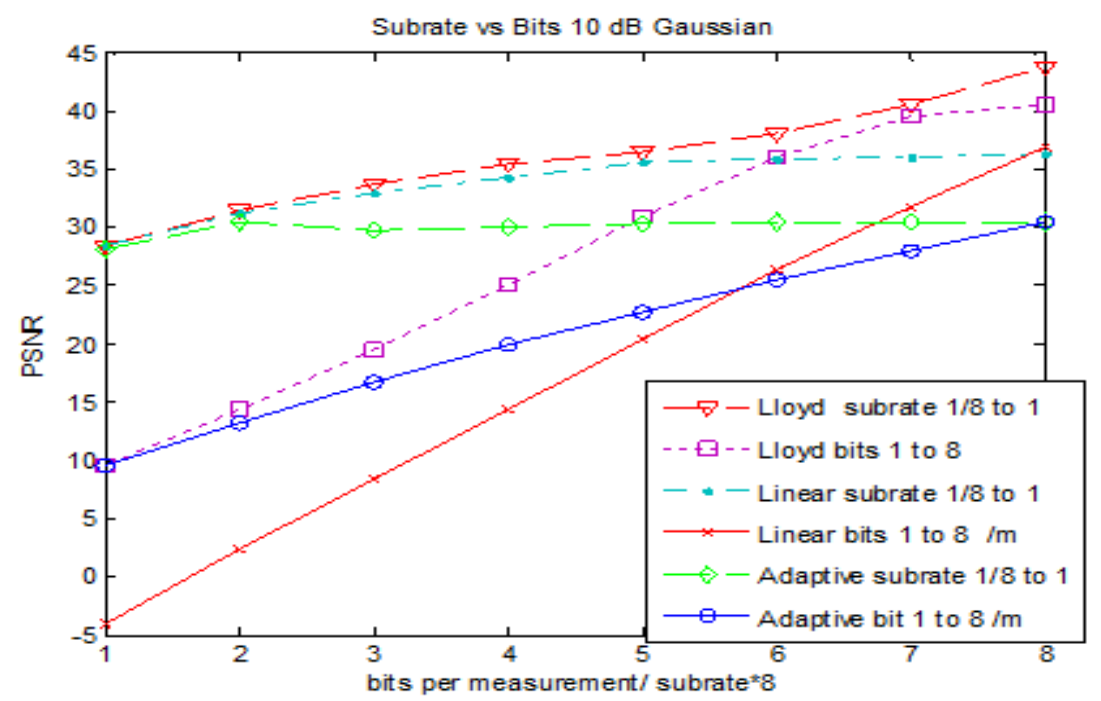

Figure-12. Subrates vs. bits in 10dB Gaussian Channel

Figures-11 and -12 show the comparison of the two approaches. It shows that, from the above results, using less measurement is a better approach than just reduce bits/measurements. However, in the system, we implement both.

\section{E. Bit Sensitivity}

In some scenario, when the wireless channel is not solid, it will be preferable to get an overview of the image first, and then get more details if possible. This is the advantage of CS comparing to other compression standards, e.g. JPEG, in CS, each of the measurements is independent. Then we can first transmit the $1^{\text {st }}$ bit of each measurements, followed by $2^{\text {nd }}, 3^{\text {rd }} \ldots$ In this scenario, even if the wireless communication has been terminated involuntarily, at the receiver side, at least it get the overview of the whole image. Figure-13 shows the reconstructed image from only the first 1 bit, 2 bits, ..., and 8 bits of each measurement.
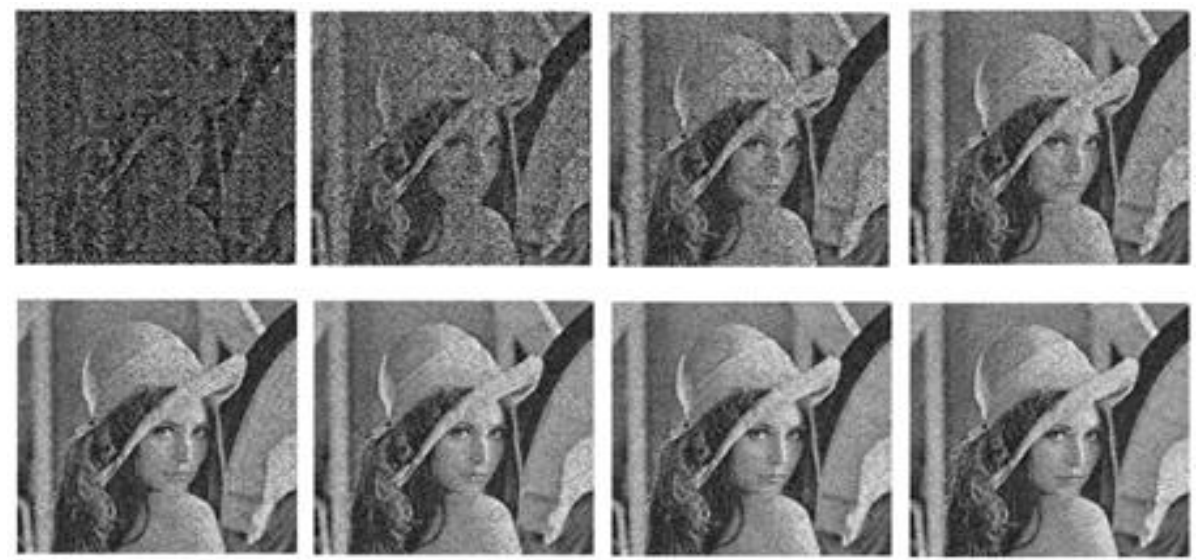

Figure-13. 5db Gaussian channel, from left to right: 1bit/measurement to 4 bit/measurement; 5bit/measurement to $8 \mathrm{bit} /$ measurement PSNR: $9.369012 .788415 .611317 .7277 ; 19.1534$ 20.197620 .600720 .9682

It is observed from Figure-13 that reconstructed images gradually getting better as more bits received, and with 2 bits/measurement, we at least get a general overview of the whole image.

\section{F. CS vs. DCT}

DCT is the core function of JPEG. The JPEG compression standard performs better for wired communication channels in which average bit error rates are very low. Wireless communication channels are characterized by higher average bit error rates, so traditional data compression methods may not perform well in wireless communication channel. Since DCT in JPEG is based on 8 by 8 blocks and the high compression rate also come from the precision of DC coefficients per block [2]. In high bit error rate wireless channel, DC coefficient and the segments between blocks may experience error transmission, which severely downgrade the reconstructed image quality.

CS based compression scheme features a linear transformation with a random orthogonal matrix. Each measurement is independent, and can be recovered individually. Any package is equally weighted. Losing any one of them will not lead to dramatic downgrade of the reconstructed signal. Here, two advantages of CS over DCT will be discussed.

\section{1) Encoding Time}

As described in the Section II, CS measurement is obtained by vector $y=\phi x$. Thus CS encoding is a very simple matrix operation. However, JPEG have many steps on encoding, including block splitting, DCT, Entropy coding, Zigzag. Thus JPEG will take much more time at encoder side than CS. Refer to figure-14, for 'Lenna', CS encoder took about 0.01-0.04s running in a Dell Precision laptop, and JPEG took about 0.5 seconds, 10-50 times more than CS. 


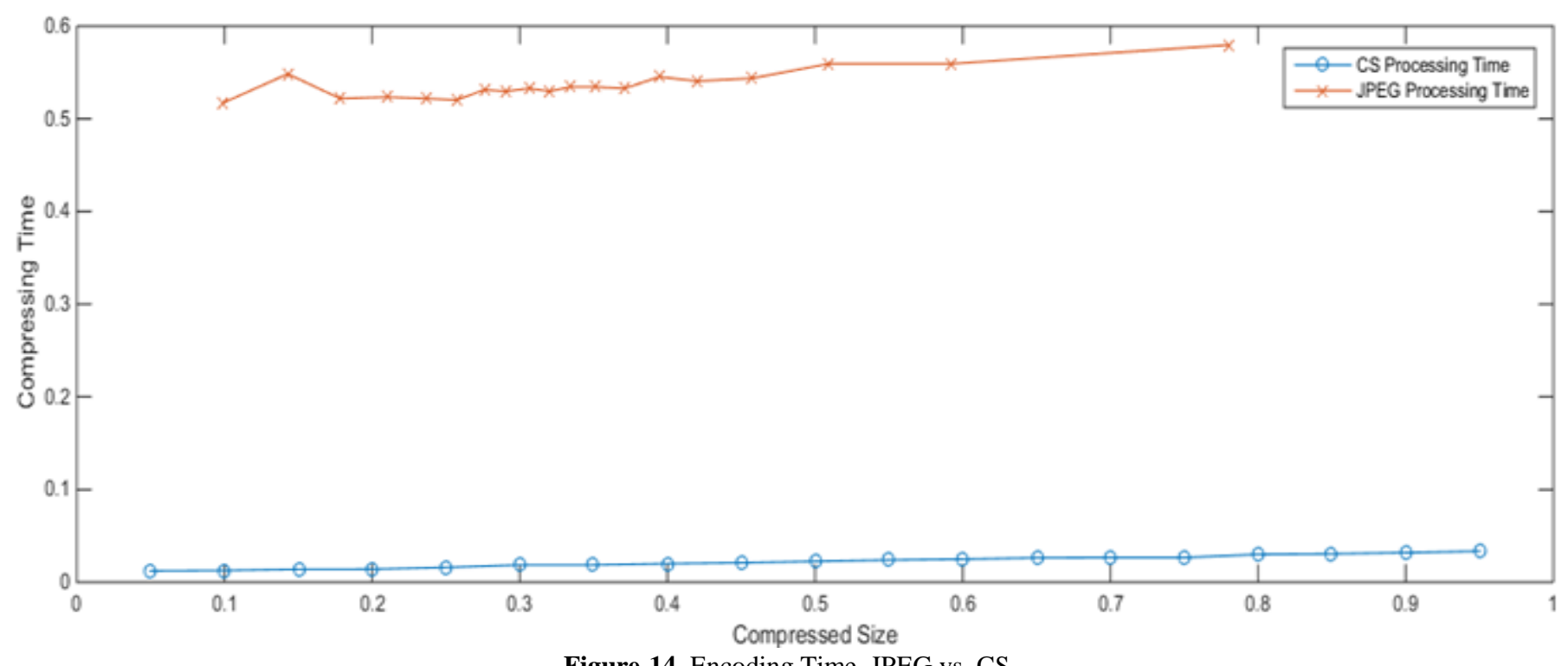

Figure-14. Encoding Time, JPEG vs. CS

Thus, CS may be implemented on platforms which requires faster encoding, e.g., camera, satellite, cellphones, and etc.

\section{2) Error Tolerance}

Since JPEG is based on 8 by 8 block splitting, during the decoding process, how to decide block splitting is critical. After noisy wireless channel, the marks of block splitting may get error thus the recovered image may got altered like Figure-15 below:
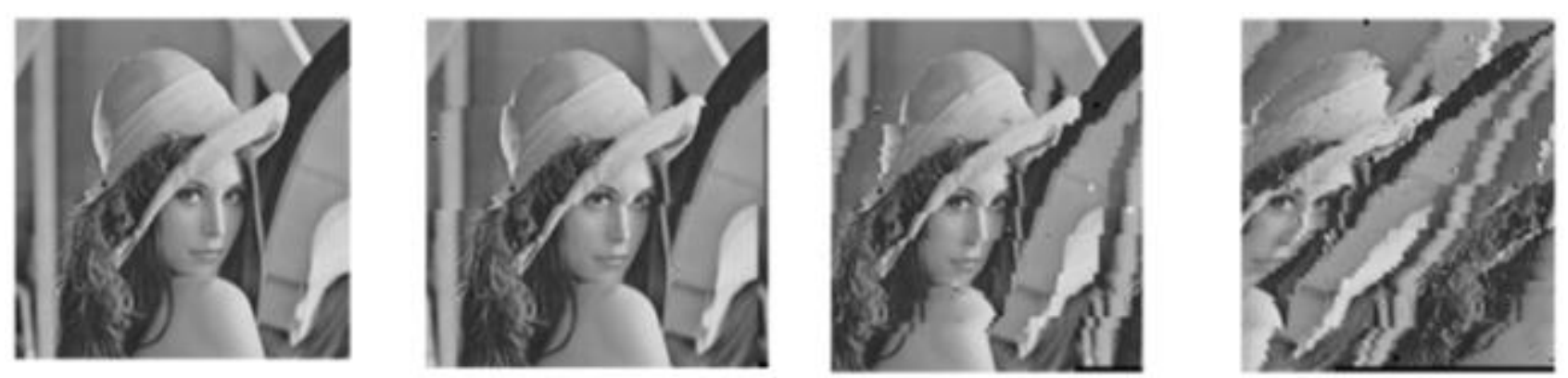

Figure-15. JPEG after noisy Gaussian Channel, 10-7 db

For CS, as shown in Figure-13, the error to each measurement would not further influence other measurements and thus CS would have better error tolerance performance at high bit error rate wireless channels.

\section{G. Performance over Multipath Fading Channel}

In previous sections, a simple Gaussian noisy channel was chosen to represent the network condition, while more complicated network models can test our proposed method in expanded application conditions.

\section{1) Two Types of Fading Channels}

Usually, multipath Rician Fading Channel is used to model multipath fading channel, which have direct line-ofsight path from transmitter to receiver and multipath Rayleigh Fading Channel is used to model multipath fading channel which have one or more major reflected paths from transmitter to receiver. Please refer to Figure-16.

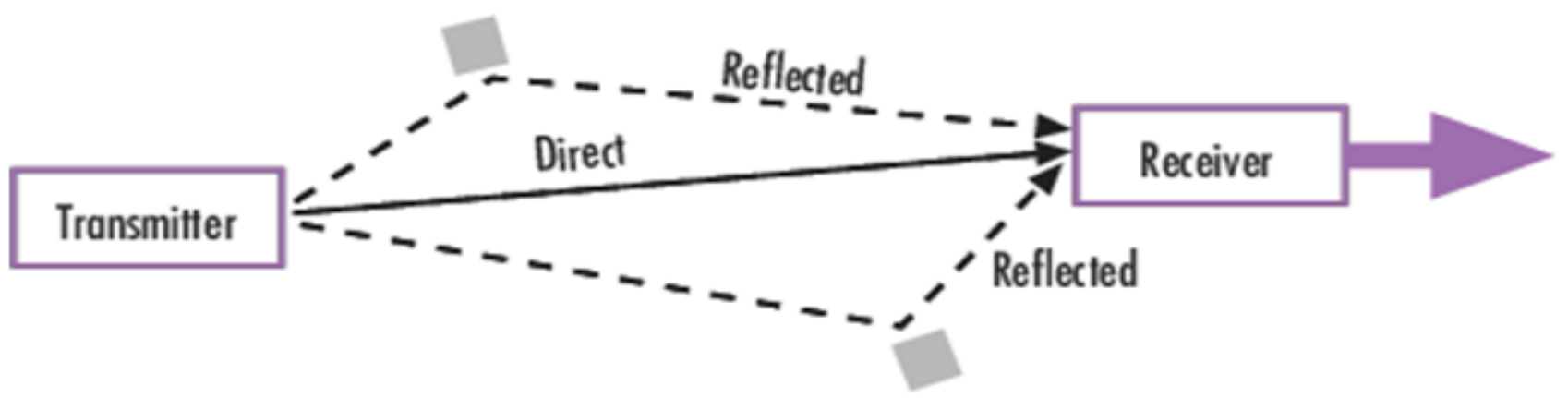

Figure-16. Fading channels of direct path and reflected paths

Source: https://www.mathworks.com/help/comm/ug/fading-channels.html

\section{2) Character of Fading Channel}

Comparing to additive white Gaussian noise (AWGN) channel, fading channels have much higher BER:

$$
\text { For Rayleigh Fading, } P_{b}=\frac{1}{2} \sqrt{1-\frac{E b / N o}{2+E b / N o}}
$$

For Rican Channel, the BER depends on the $k$ factor, which is the ratio between the power in the direct path and the power in the other scattered paths. Theoretical BER for BPSK over Rician Fading Channel with AWGN noise is 
given by the following equation:

$$
P_{b}=\frac{1}{2} e r f c \sqrt{\frac{K * E b / N o}{K+E b / N o}}
$$

Please refer to Figure-17 for the BER.

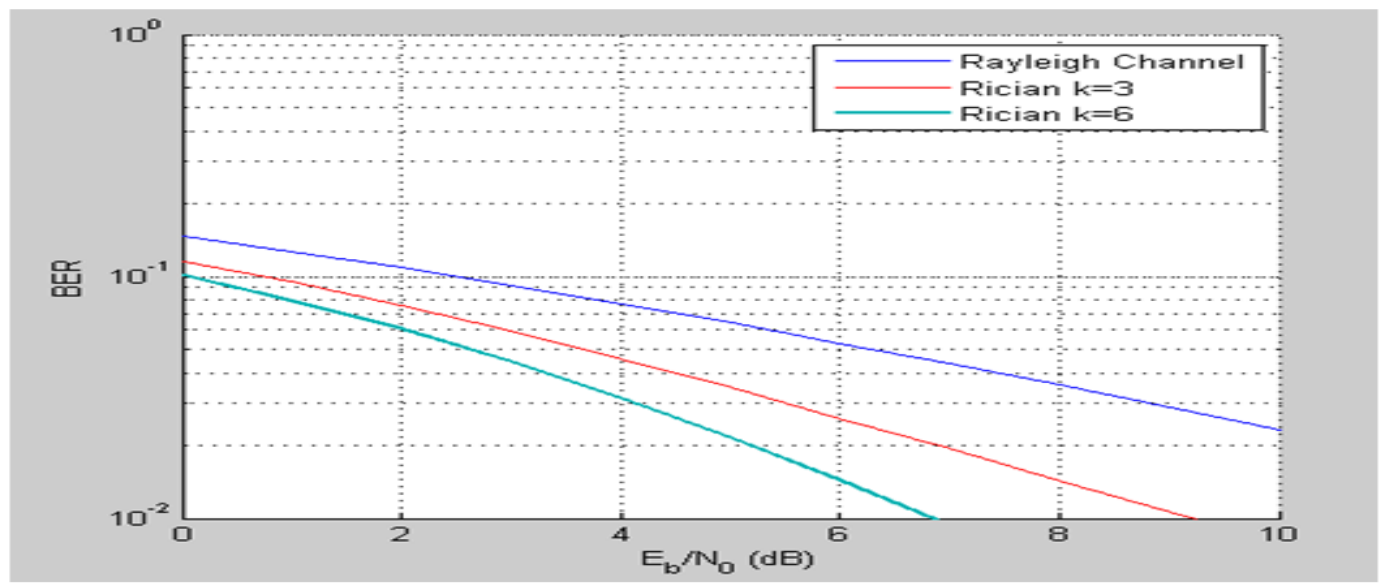

Figure-17. BER for Rayleigh Channel and Rician Channel

\section{3) Wireless Transmission Strategy in Fading Channels}

Given a fading channel, our proposed system has following controllable factors which can impact the results:

i: subrate

ii: bits per measurement and power adjustment

iii: quantizer

iv: block size

Similar to the discussion on AWGN, optimization is considered among the best choices of those parameters.

a) Best subrate to be used in fading channel:

(1) The following images in Figure-18 show at Rayleigh Channel with SNR=10dB, block size=32, using 8bits/ measurement, the recovered image at subrate $=0.01,0.06,0.11,0.16,0.3,0.5,0.7$ and 1 from left to right.

We can conclude that there is no significant difference when subrate $>0.1$. The Simulation results can validate this conclusion.
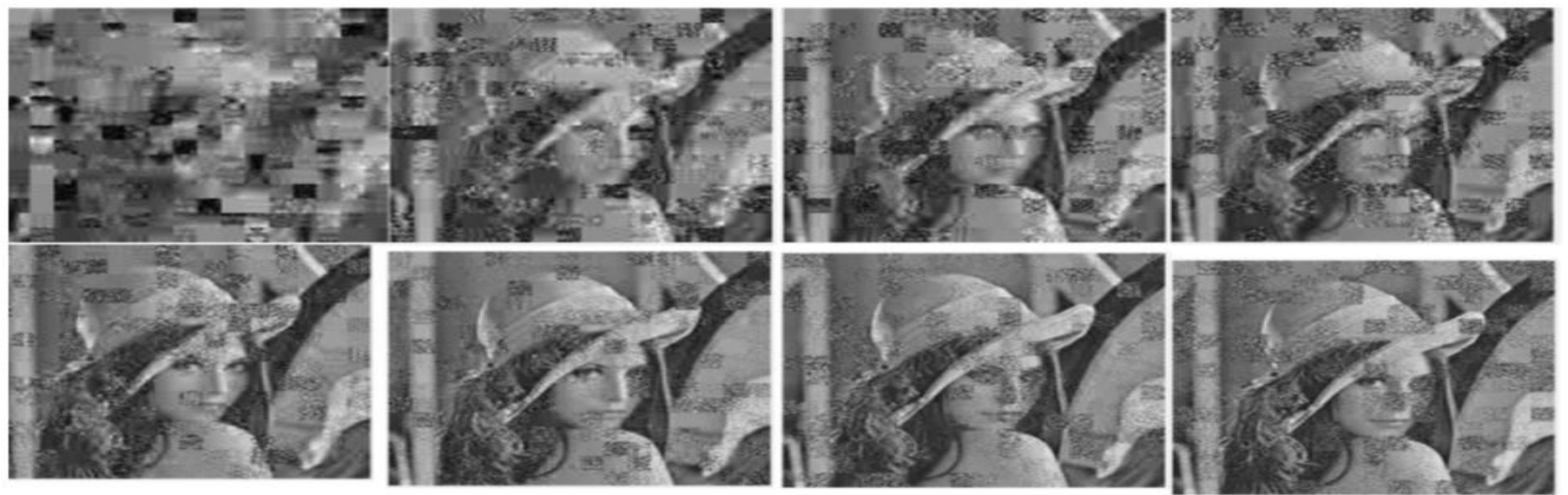

Figure-18. Reconstructed images under Rayleigh Channel

(2) With same block size, quantizater, bits per measurement, and various subrate under various channel SNR condition, the reconstructed PSNR for images Lenna, Goldhill, and Barbara, are illustrated in Figures -19, -20, and 21 , respectively.

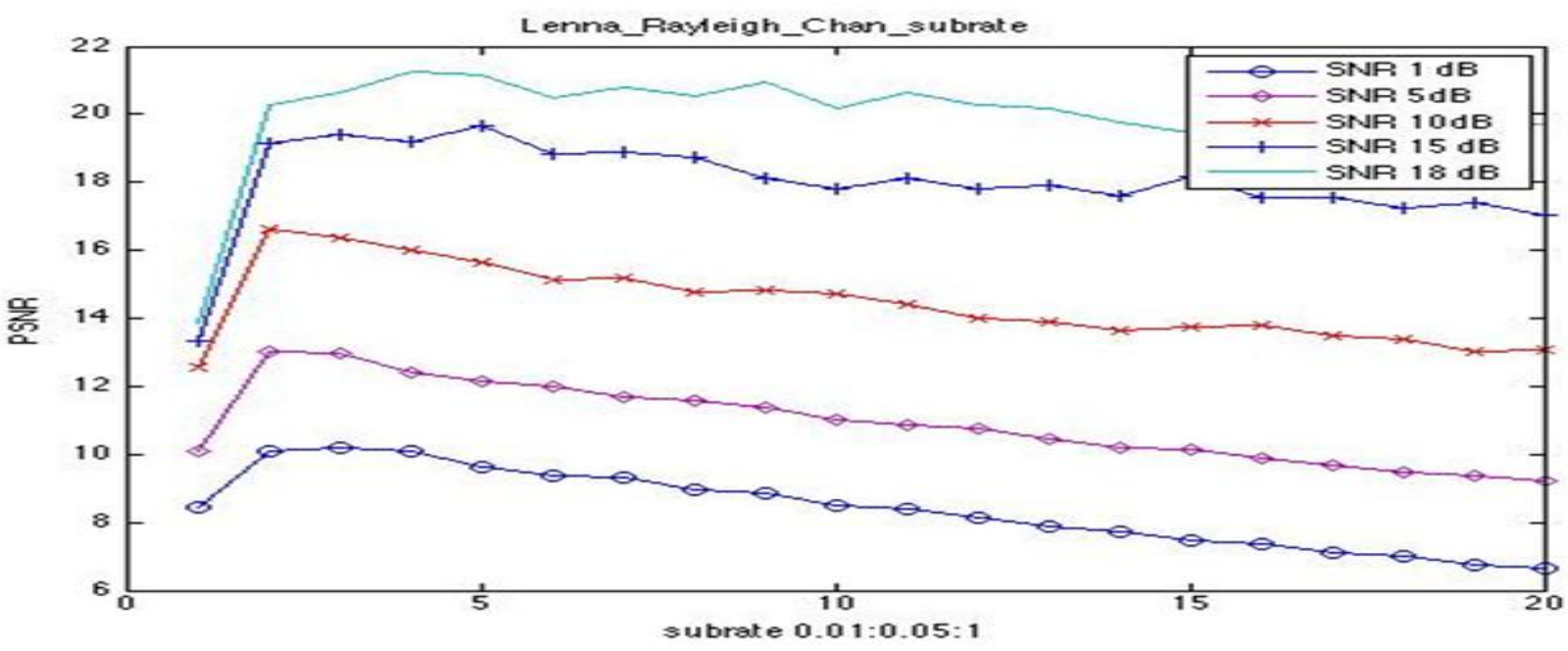

Figure-19. PSNR for reconstructed Lenna over Rayleigh Channel 


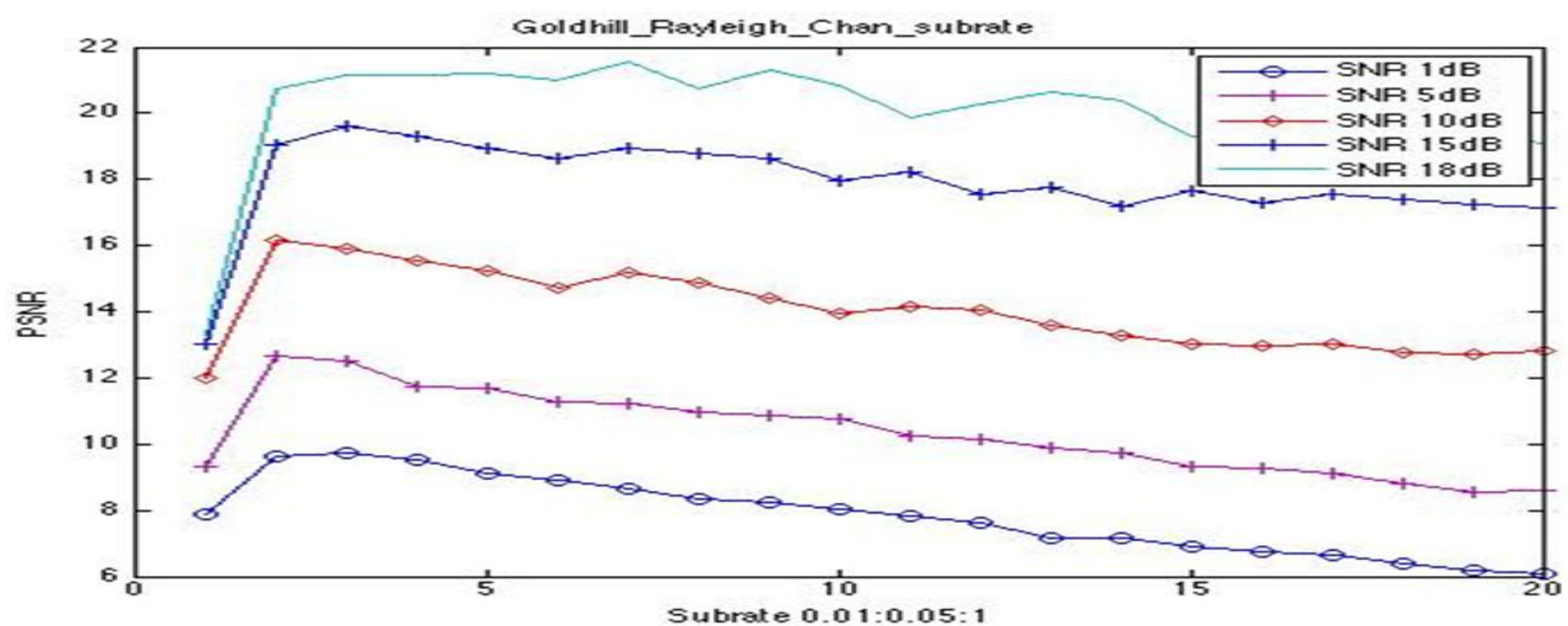

Figure-20. PSNR for reconstructed Goldhill over Rayleigh Channel

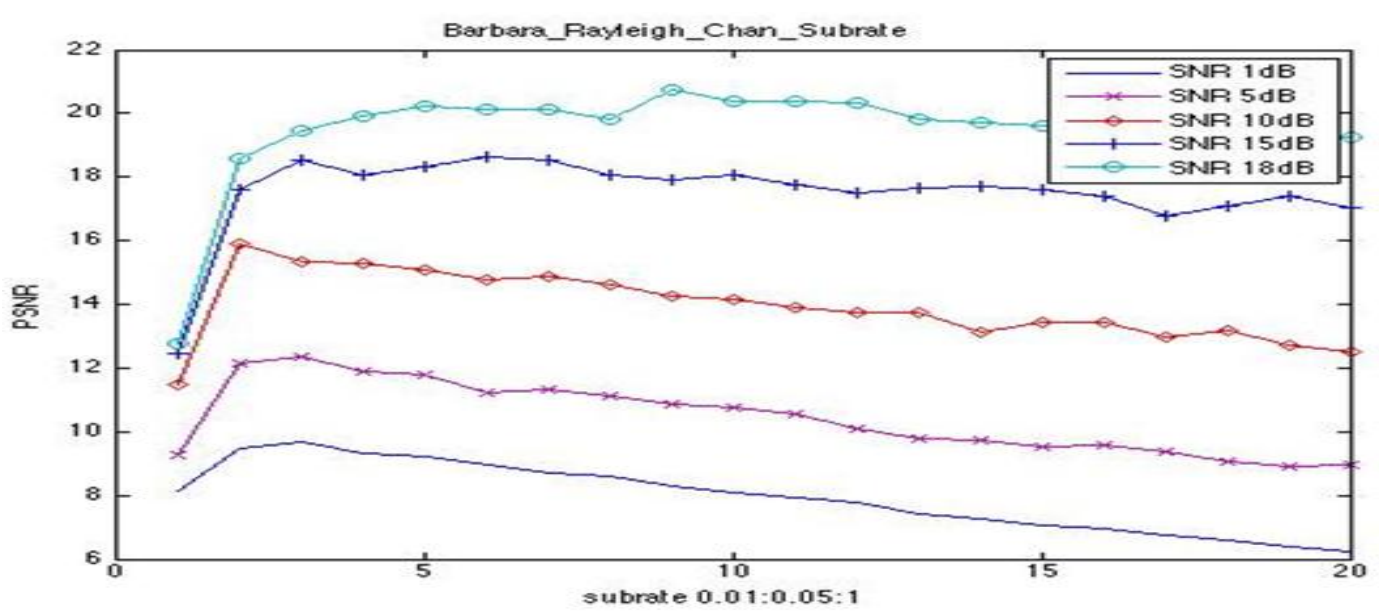

Figure-21. PSNR for reconstructed Barbara over Rayleigh Channel

From above simulation results, we can conclude that in Rayleigh Channel, the optimized subrate is 0.06-0.11. (3) Same experiment is conducted for Rician channel. Simulation results of optimized subrate are presented in Figures -22 through -24 .

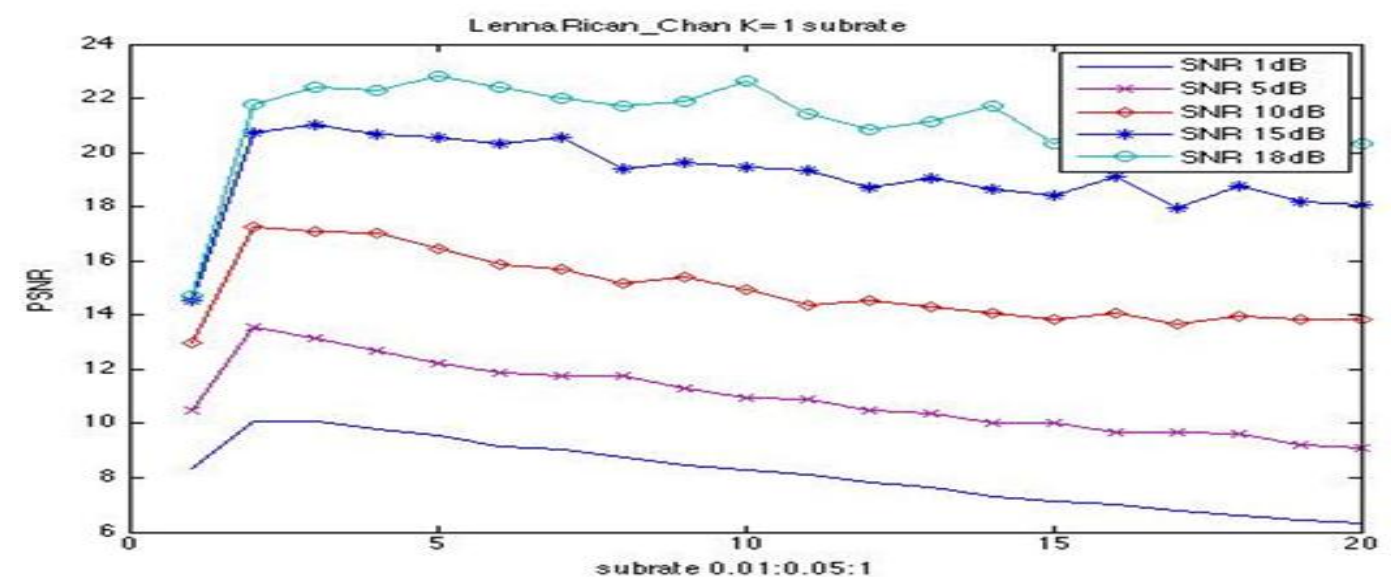

Figure-22. PSNR for reconstructed Lenna over Rician channel $(\mathrm{K}=1)$

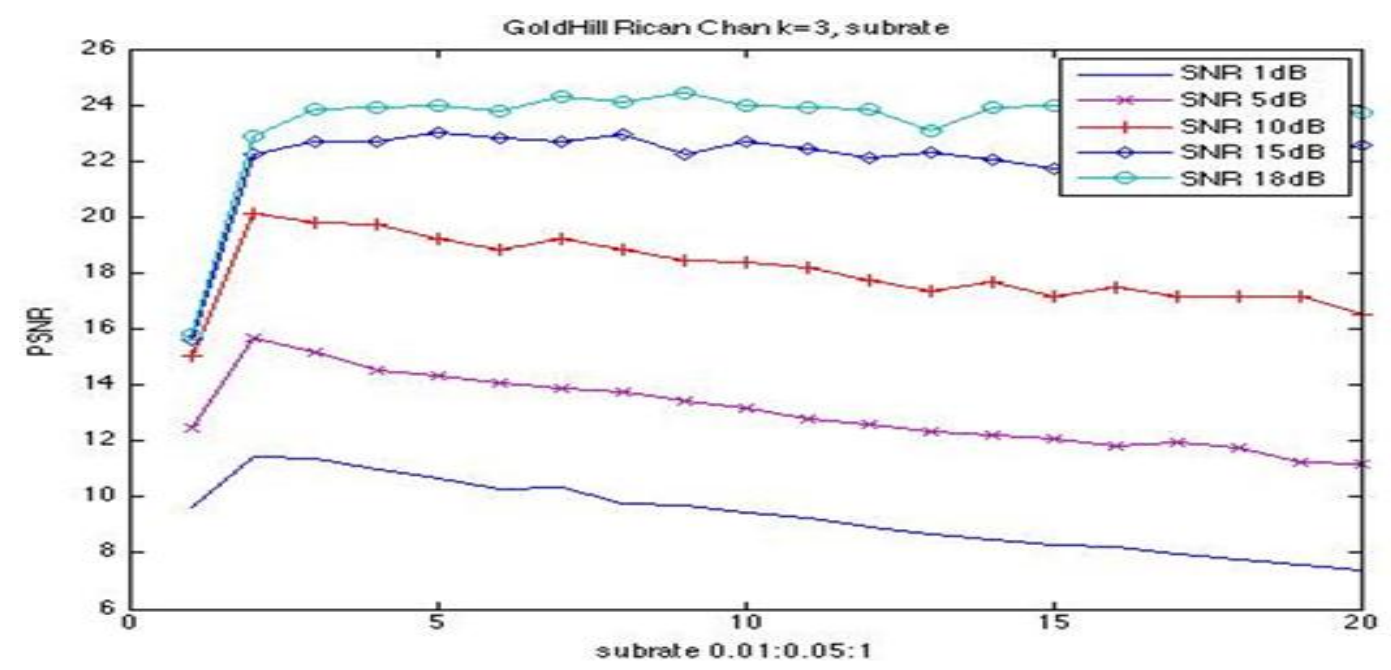

Figure-23. PSNR for reconstructed Goldhill over Rician channel $(\mathrm{K}=3)$ 


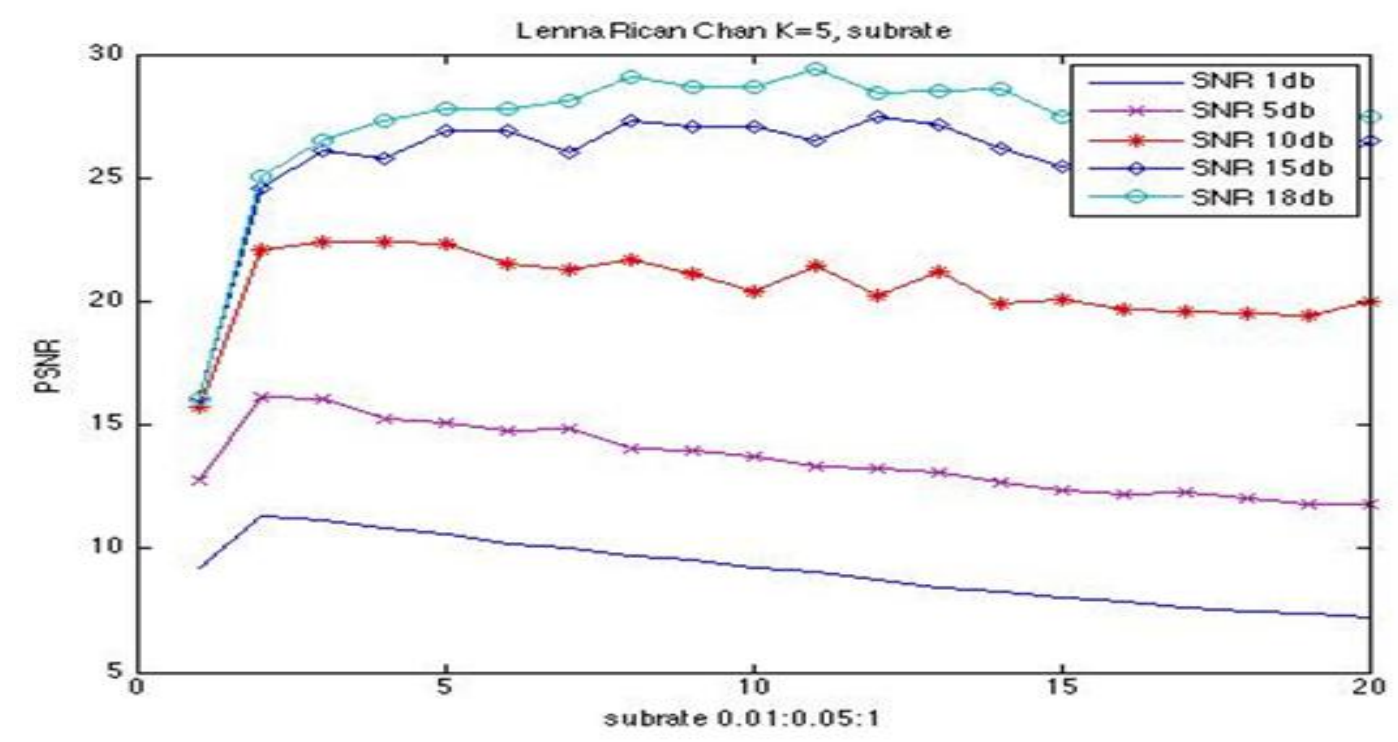

Figure-24. PSNR for reconstructed Lenna over Rician channel (K=5)

From experimental results, the optimized subrates in fading channel are 0.06 (low SNR channel) and 0.11 (high SNR channel).

b) Optimized bits per measurement:

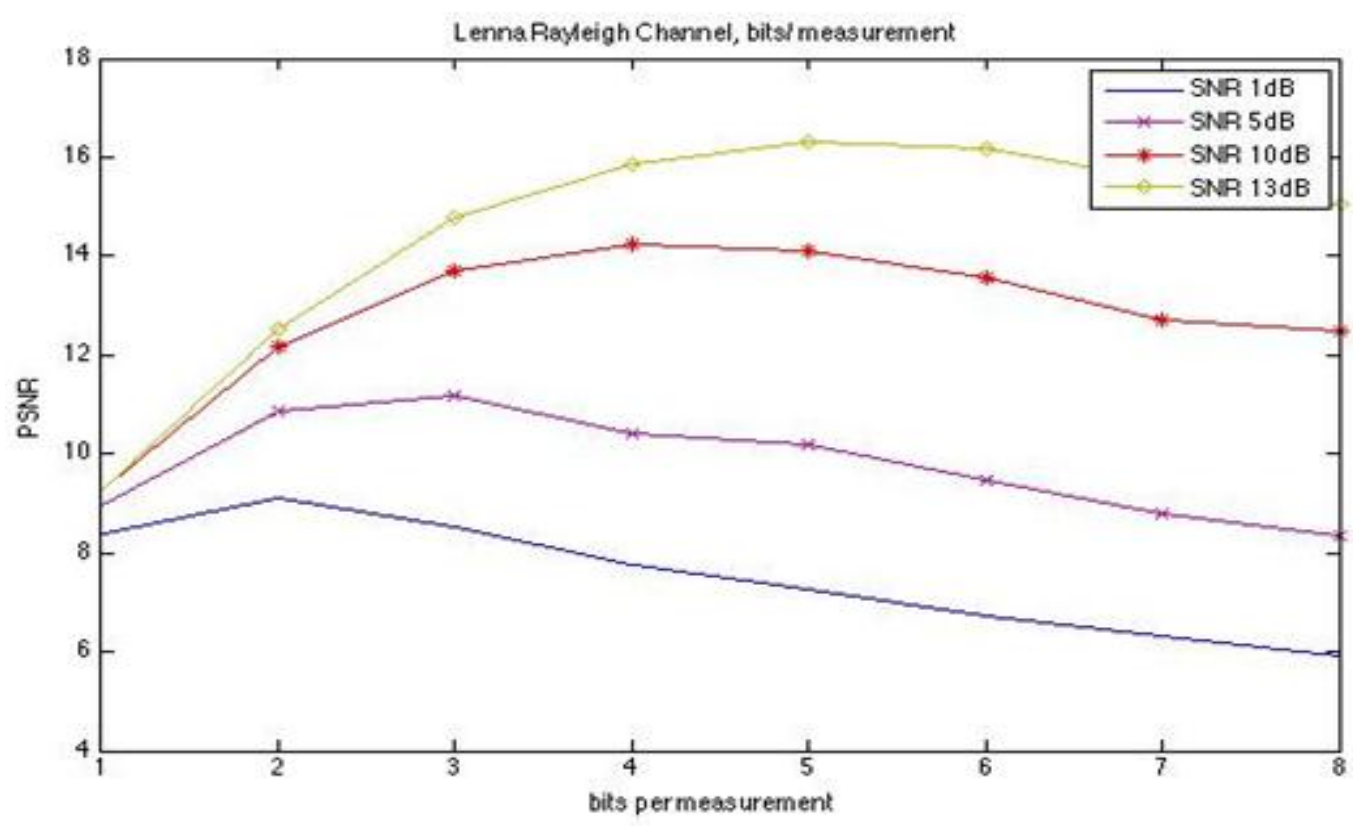

Figure-25. PSNR after adjusted power per bit in Rayleigh Channel

Similar to Table-2, refer to Figure-25, in Fading Channel, we also tabulate the optimized bits/ measurement in Rayleigh Channel in Table-3.

Table-3. Optimized Bits per Measurement in Different Rician Channel Snr

\begin{tabular}{l|l}
\hline \multicolumn{2}{c}{ Table-3. Optimized Bits per Measurement in Different Rician Channel Snr } \\
\hline SNR (dB) & Optimal bits/measurement \\
\hline 1 & 2 \\
\hline 2 & 2 \\
\hline 3 & 2 \\
\hline 4 & 2 \\
\hline 5 & 3 \\
\hline 6 & 3 \\
\hline 7 & 3 \\
\hline 8 & 3 \\
\hline 9 & 3 \\
\hline 10 & 4 \\
\hline 11 & 4 \\
\hline 12 & 4 \\
\hline 13 & 5 \\
\hline
\end{tabular}

\section{Conclusion}

In this paper, we investigate the CS based image compression and transmission over wireless channel. We introduced a CDF based quantizer and showed that it performs better than Lloyd and linear quantizer in noisy channel. Then we further discussed optimal image transmission through noisy channel by using different bits/measurement and subrates according to the channel's condition. We finally compared CS to JPEG and found that CS performed better. Not only Gaussian channel, but also Rayleigh and Rician fading channel conditions are considered in optimizing the 
parameters for CS based scheme. For further research, we would develop more solid mathematical based research on this issue, and also explore compressive sensing based video compression and transmission via noisy channel.

\section{References}

[1] S. Morgan, The Mobile Internet Report, 2009.

[2] International Telegraph and Telephone Consultative Committee, "Digital compression and coding of continuous-tone still images." JPEG standard (JPEG ISO/IEC 10918-1 ITU-T Recommendation T.81), 1992.

[3] K. R. Rao and P. Yip, Discrete cosine transforms - algorithms, advantages, applications. London: Academic Press Inc, 1990.

[4] A. Boukerche and Y. Hu, "QoS- based JPEG images transmission protocol for wireless CDMA communication systems," in Proceedings of the 1st ACM Workshop on Wireless Multimedia Networking and Performance Modeling, Montreal, Canada, October 2005, 2005, pp. 38-45.

[5] E. Candès and T. Terence, "Near-optimal signal recovery from random projections: Universal encoding strategies?," IEEE Transactions on Information Theory, vol. 52, pp. 5406 - 5425, 2006. View at Google Scholar | View at Publisher

[6] D. Donoho, "Compressed sensing," IEEE Transactions on Information Theory, vol. 52, pp. 1289 - 1306, 2006. View at Google Scholar

[7] V. K. Goyal, A. K. Fletcher, and R. Sundeep, "Compressive samplingand lossy compression." IEEE Signal Processing Magazine, vol. 25, pp. 48-56, 2008. View at Google Scholar $\mid$ View at Publisher

[8] N. Ahmed, T. Natarajan, and R. R. Kamisetty, "Discrete cosine transform," IEEE Transactions on Computers, vol. 100, pp. 90-93, 1974. View at Google Scholar

[9] Y. Zhang, S. Cui, and D. R. Vaman, "Compressive sensing based optimized image transmission over wireless Gaussian channel," presented at the 2nd International Conference on Intellectual Technique in Industrial Practice, Changsha China, 2010.

[10] J. E. Fowler, S. Mun, and E. W. Tramel, "Block-based compressed sensing of images and video," Foundations and Trends in Signal Processing, vol. 4, pp. 297-416, 2012. View at Google Scholar

[11] S. Lloyd, "Least squares quantization in PCM," IEEE Transactions on Information Theory, vol. IT-28, pp. 129-137, 1982. View at Google Scholar | View at Publisher 